\title{
Fanconi-Bickel Syndrome - Mutation in SLC2A2 Gene
}

\author{
Mohit Kehar • Sunita Bijarnia • Sian Ellard • \\ Jayne Houghton • Renu Saxena • I. C. Verma • \\ Nishant Wadhwa
}

Received: 29 November 2013 / Accepted: 2 May 2014 / Published online: 10 June 2014

(C) Dr. K C Chaudhuri Foundation 2014

\begin{abstract}
Fanconi-Bickel Syndrome (FBS) is a rare autosomal recessive disorder of carbohydrate metabolism. The defect in the GLUT 2 receptors in the hepatocytes, pancreas and renal tubules leads to symptoms secondary to glycogen storage, glucose metabolism and renal tubular dysfunction. Derangement in glucose metabolism is classical with fasting hypoglycemia and post-prandial hyperglycemia. The authors report a 4-year-old boy who presented with failure to thrive, motor delay, protuberant abdomen and was noted to have huge hepatomegaly with glycogen deposition in liver, and renal tubular acidosis. Gene sequencing revealed homozygous mutation, c.1330T > C in SLC2A2 gene, thus confirming the diagnosis of FBS. Only three mutations have been reported from India so far. The primary reason for referral to authors' hospital was for liver transplantation, but an accurate diagnosis led to avoidance of the major surgery and streamlining of treatment with clinical benefit to the child and family.
\end{abstract}

Keywords Fanconi-Bickel syndrome $\cdot$ Renal tubular acidosis $\cdot$ Hyperglycemia $\cdot$ Glycogenolysis $\cdot S L C 2 A 2$ gene $\cdot$ India

\footnotetext{
M. Kehar $\cdot$ N. Wadhwa

Division of Pediatric Gastroenterology and Hepatology, Sir Ganga Ram Hospital, Rajendra Nagar, New Delhi, India

S. Bijarnia $(\triangle) \cdot$ R. Saxena $\cdot$ I. C. Verma

Center of Medical Genetics, Sir Ganga Ram Hospital, Rajendra

Nagar, New Delhi 110060, India

e-mail: Bijarnia@gmail.com

S. Ellard $\cdot$ J. Houghton

Department of Molecular Genetics, Royal Devon and Exeter NHS

Foundation Trust, Exeter EX2 5AD, UK

S. Ellard

Institute of Biomedical and Clinical Science, University of Exeter Medical School, Exeter EX2 5DW, UK
}

\section{Introduction}

Fanconi-Bickel Syndrome (FBS), a rare genetic disorder of carbohydrate metabolism, was first described by Fanconi and Bickel in 1949 [1]. The authors report a case of FBS presenting at $4 \mathrm{y}$ of age whose correct diagnosis led to avoidance of the liver transplant.

\section{Case Report}

A 4-year-old boy, born to third degree consanguineous couple (first cousins), from Maharashtra presented with failure to thrive, delayed mile stones and progressive abdominal distention since infancy. There was no history of seizures or jaundice. The child had cherubic facies, height of $81 \mathrm{~cm}(<3 \mathrm{rd}$ percentile) and weight $10.8 \mathrm{~kg}(<3 \mathrm{rd}$ percentile). Features of active rickets (wrist widening, frontal bossing and hypotonia) were present (Fig. 1). His developmental age corresponded to $2 \mathrm{y}$. Soft hepatomegaly was observed with liver span of $15 \mathrm{~cm}$; no splenomegaly or evidence of ascites was noted. Systemic examination was otherwise unremarkable. Investigations revealed normal blood counts, including absolute neutrophils, metabolic acidosis ( $\mathrm{pH} 7.23$, bicarbonate $16.9 \mathrm{mmol} / \mathrm{L})$, hyperchloremia $(113.9 \mathrm{mmol} / \mathrm{L})$ and normal anion gap. GSD Ia was ruled out after gene sequencing. Evidence of proximal renal tubular acidosis (RTA) was noted (generalized aminoaciduria on thin layer chromatography, glucosuria +++ and proteinuria ++ ). Total cholesterol was $249 \mathrm{mg} / \mathrm{dl}$ (normal: <195) and triglycerides were $681 \mathrm{mg} / \mathrm{dl}$ $(<145)$. Post-prandial and post glucose hyperglycemia was documented on two occasions $(246 \mathrm{mg} / \mathrm{dl}$ and $252 \mathrm{mg} / \mathrm{dl})$ after episode of hypoglycemia $(40 \mathrm{mg} / \mathrm{dl})$. Fasting plasma lactate was $10.2 \mathrm{mg} / \mathrm{dl}(4.5-20 \mathrm{mg} / \mathrm{dl})$. Liver function tests (serum proteins, bilirubin, ALT, AST) including prothrombin time were normal. Blood urea nitrogen, creatinine and uric acid were normal (10, 0.6 and $2.9 \mathrm{mg} / \mathrm{dl}$, respectively). Serum calcium 


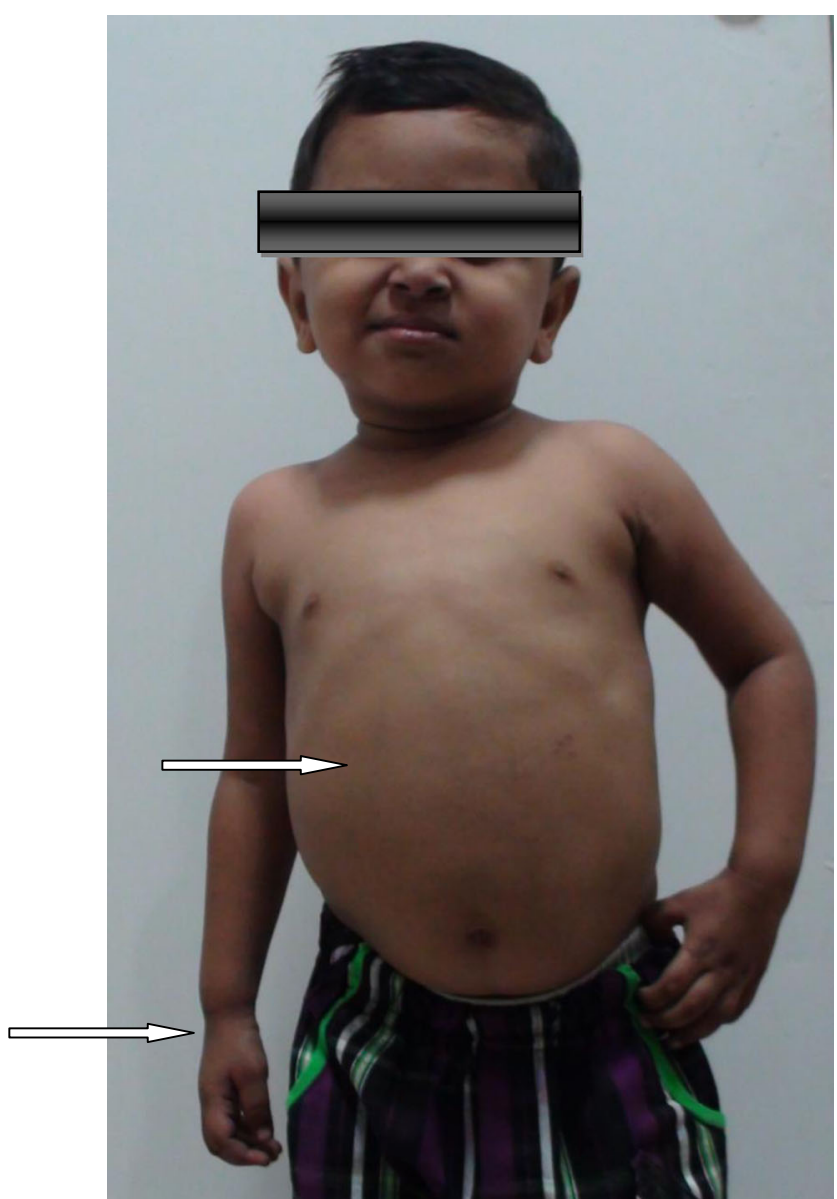

Fig. 1 Patient with cherubic facies, protuberant abdomen and wrist widening

was $8.5 \mathrm{mg} / \mathrm{dl}$, phosphorus was $2.0 \mathrm{mg} / \mathrm{dl}(2.5-4.6)$ and alkaline phosphatase level was high 1044 (117-390). Wrist radiograph showed features of rickets. His abdominal ultrasonography revealed hepatomegaly with coarse echotexture. Liver biopsy showed accumulation of glycogen in hepatocytes with no disturbance in liver architecture. In view of features of RTA, rickets, hepatomegaly, fasting hypoglycemia and postprandial hyperglycemia, glycogen accumulation in liver, a diagnosis of FBS was suspected. Sanger sequence analysis was performed for $S L C 2 A 2$ gene, which revealed a previously reported homozygous mutation, c.1330 $>\mathrm{C}$ in exon 10, resulting in change of amino acid from Tryptophan to Arginine at 444 amino acid position (p.Trp444Arg), thus confirming the diagnosis of FBS (Fig. 2). After establishment of diagnosis, the family was counseled regarding the conservative management and liver transplant was avoided. Dietary management was started with uncooked corn starch and elimination of lactose in diet. Supplementation with Vitamin D, phosphorus, and bicarbonate were added. On follow up after 3 mo the child showed improvement in rickets and gain of weight and height (weight $12.2 \mathrm{~kg}$ and height $83 \mathrm{~cm}$ ), however with persistence of aminoaciduria.

\section{Discussion}

FBS is a rare disease of carbohydrate metabolism (previously termed as glycogen storage disease type XI) occurring due to pathogenic mutations in GLUT 2 transporter gene, SLC2A2. Genetic defect in GLUT2 was proposed as the possible metabolic basis for FBS by Santer et al. in 1997 [2].

GLUT2 is amongst family of monosaccharide transporters that transport sugars in an energy-independent manner. GLUT2 transports glucose and galactose into hepatocytes after feeding and exports free glucose out of hepatocytes during fasting [2]. The hyperglycemia and hypergalactosemia

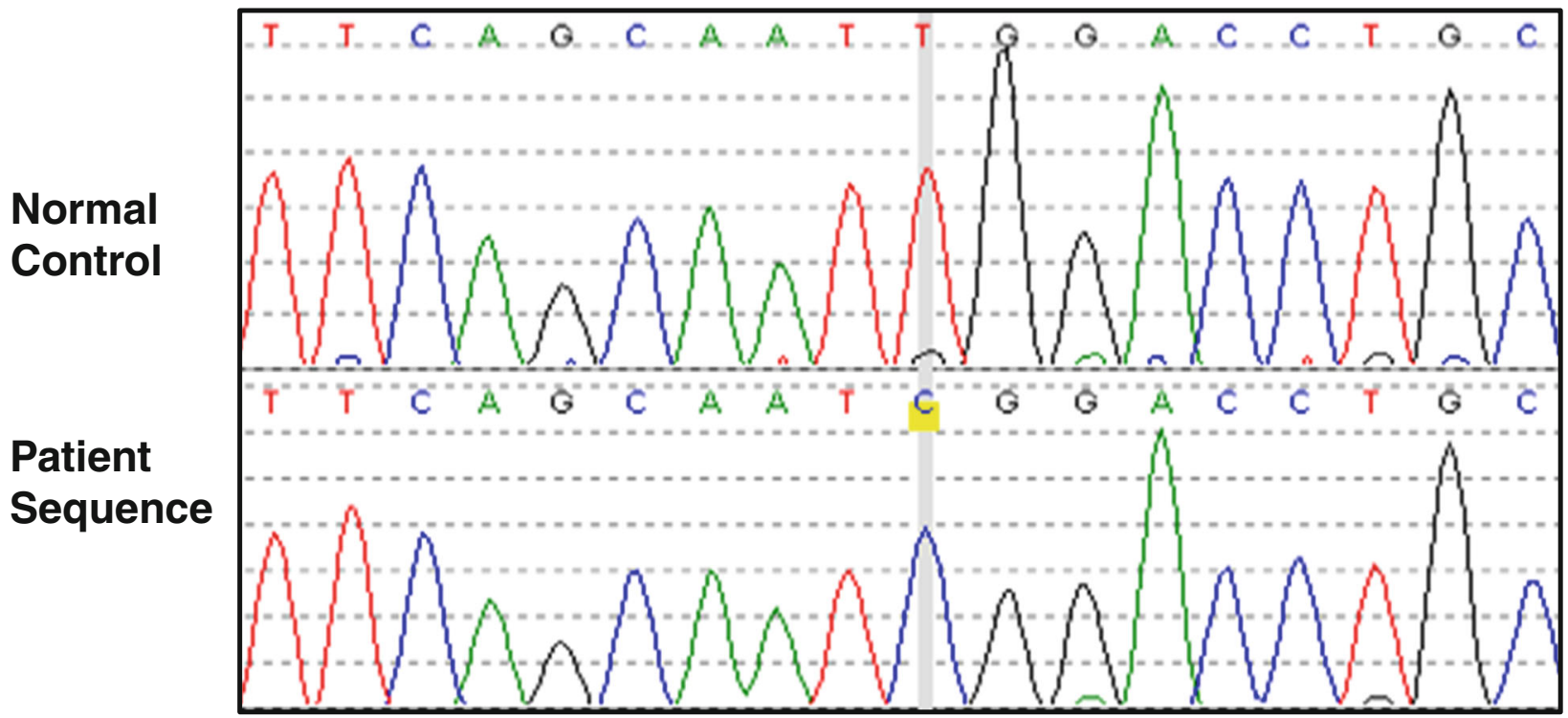

Fig. 2 Sequence chromatogram showing single homozygous base pair change, c.1330T $>\mathrm{C}$ (p.W444R) in $S L C 2 A 2$ gene 
seen in postprandial state are due to reduced uptake of these monosaccharides by the liver and may be enhanced by the poor insulin response to elevated blood glucose levels demonstrated in patients with FBS. Fasting hypoglycemia results from defective export of free glucose from hepatocytes when peripheral glucose supplies have been exhausted [2]. Glycosuria is the result of failure to export glucose across the basolateral membranes of renal tubular cells.

Patients of FBS typically present with combination of clinical symptoms: hepatomegaly secondary to glycogen accumulation, glucose and galactose intolerance, fasting hypoglycemia, a characteristic tubular nephropathy, and severely stunted growth [3]. Patients presenting late develop cherubic face, truncal obesity, retarded growth and puberty, bone problems associated with hypophosphatemic rickets, and dental caries.

There is no specific treatment of FBS, the management for renal Fanconi syndrome includes management of RTA with maintenance of water and electrolyte balance; supplementation of vitamin $\mathrm{D}$, calcium, phosphorus, and bicarbonate. Small frequent meals with uncooked cornstarch, is advocated [4]. The prognosis for this condition appears to be generally good in terms of survival, but these patients are universally short in stature. Liver transplant is not required for management of patients suffering from FBS.

FBS has only been reported in a few cases from India, all from consanguineous families presenting similarly with hepatomegaly and renal dysfunction [5-8]. All mutations have been shown to be different, thus showing lack of any common mutation in India [6-8]. Genetic counseling is an integral part of management in view of autosomal recessive nature of the condition and $25 \%$ risk of recurrence in siblings. With the knowledge of mutations prenatal diagnosis can be offered to couples in subsequent pregnancies, using mutation analysis on chorionic villous sampling at $11 \mathrm{wk}$ of pregnancy.
Acknowledgments SE is a Wellcome Trust Senior Investigator; the authors acknowledge the contribution of Wellcome Trust for making this study possible.

Contributions MK and SB: Wrote the manuscript; SE and JH: Carried out molecular genetic testing for FBS; RS: Involved in molecular testing; SB, NW and ICV: Involved in management of case; ICV: Provided critical comments. SB will act as guarantor for this paper.

Conflict of Interest None.

Source of Funding Wellcome trust provided funds for molecular genetic study.

\section{References}

1. Fanconi G, Bickel H. Die chronische Aminoaciduria (Aminosaeurediabetes oder nephrotisch-glukosurischer Zwergwuchs) bei der Glykogenose und der Cystinkrankheit. Helv Paediat Acta. 1949;4:359-96.

2. Santer R, Schneppenheim R, Dombrowski A, Götze H, Steinmann B, Schaub J. Mutations in GLUT2, the gene for the liver-type glucose transporter, in patients with Fanconi-Bickel syndrome. Nat Genet. 1997; 17:324-6.

3. Santer R, Steinmann B, Schaub J. Fanconi-Bickel syndrome-a congenital defect of facilitative glucose transport. Curr Mol Med. 2002;2:213-27.

4. Lee PJ, Van't Hoff WG, Leonard JV. Catch-up growth in FanconiBickel syndrome with uncooked cornstarch. J Inherit Metab Dis. 1995;18:153-6.

5. Karande S, Kumbhare N, Kulkarni M. Fanconi-Bickel syndrome. Indian Pediatr. 2007;44:223-5.

6. Mohandas Nair K, Sakamoto O, Jagadeesh S, Nampoothiri S. Fanconi-Bickel syndrome. Indian J Pediatr. 2012;79:112-4.

7. Gopalakrishnan A, Kumar M, Krishnamurthy S, Sakamoto O, Srinivasan S. Fanconi-Bickel syndrome in a 3-year-old Indian boy with a novel mutation in the GLUT2 gene. Clin Exp Nephrol. 2011;15:745-8.

8. Ekbote AV, Mandal K, Agarwal I, Sinha R, Danda S. Fanconi- Bickel Syndrome: mutation in an Indian patient. Indian $\mathrm{J}$ Pediatr. 2012;79:810-2. 